\title{
PARS - Portale di formazione e informazione per il contrasto dell'analfabetismo religioso
}

L'articolo descrive il progetto Parma in tempo di guerra 1940-1945, realizzato da Istituto storico della Resistenza e dell'età contemporanea di Parma - Isrec Parma. Il progetto prevede la realizzazione di quattro portali web che raccontano - attraverso mappe, immagini, documenti e schede - i molteplici aspetti della Seconda guerra mondiale, adottando la prospettiva del Parmense. Il primo atto è Prigionieri nei campi alleati: un portale sui militari del Parmense detenuti dagli Alleati.

The article describes the project Parma in Wartime 1940-1945, led by Istituto storico della Resistenza e dell'età contemporanea di Parma - Isrec Parma. The project aims to realize four websites about several aspects of the Second World War, using maps, pictures, documents and texts, by adopting the point of view of Parma and its province. The first act of this project is POWs in the Allied Camps: a website about the Parmesan POWs held prisoners in the Allied camps.

\section{II progetto}

I militari al fronte, la prigionia, la propaganda e le mobilitazioni, la vita quotidiana, l'occupazione tedesca e la Resistenza. Adottando la prospettiva di Parma e della sua provincia, il progetto Parma in tempo di guerra 1940-1945 racconta - attraverso una serie di portali web - i molteplici aspetti della Seconda guerra mondiale, facendo ampio uso di mappe, documenti, immagini e schede di approfondimento. 
Il progetto - disponibile al link https://parmaintempodiguerra.it/ - avrà una durata triennale, prevedendo la realizzazione di quattro portali tematici e la rielaborazione della storiografia già prodotta in materia, integrandola con nuove ricerche. I contenuti sono proposti in maniera interattiva, rispettando le esigenze di un pubblico generico e, al tempo stesso, proponendosi come importante strumento didattico.

Il 3 luglio 2020 è stato presentato il primo atto questo progetto: Prigionieri nei campi alleati, disponibile al link: https://prigionieri.parmaintempodiguerra.it/

\section{Isrec Ora le memorie di 1.181 prigionieri sono storia di tutti}

Nel portale su Parma in tempo di güerra i documenti riordinati grazie all'Istituto storico della Resistenza ANTONIO BERTONCINI La memoria che corre sul persone di Parma e provincia che hanno vissuto l'esperien della prigionia in tutti $i$ contnenti, dopo aver combatturo
lontano dalla loro terra. Stor poco note di uomini dimenticati, ingiustamente guardat
con sospetto anche dopo il ritorno in patria. Fra loro c'e c ha sofferto la fame, ehi ha inparato un lavoro, chi ha trovat
lámore, chi ha avuto la dist l'amore, chi ha avuto la dist-
lusione dalla propaganda fas lusione dalla propaganda fasc-
sta, chi ha vissuto il ritorno come paura e come speranza. A riesumare lettere, sched documenti, elenchi, ha penssistenza e l'età contempo primo sito realizzato nell'a bitodel più vasto progetto $P$ ma in tempo di guert anniversario dello seconda guerra mondiale, grazie a collaborazione con Comu Provincia, Regione eFondazi gitale di public history ad alta tale di public history ad alta
ivulgaziones, lo ha definito

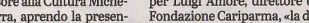
zione allauditorium Carlo (1) iennale ha il merito di renere fruibile a tutti il materiale già presente nella sede dell'Iwro di lungo periodo destiazie alla flessibilità dei nuov strumentididivulgazione. Ancor più significativo e che denlia Tondelli, il giovane ricer tore scomparso troppo presto
in circostanze tragiche, al quain circostanze tragiche, al qua esi deve lavvio dir questo nemorias. Gianpaolo Cantoni, intervenuricordi della mamma, dei ber la che illuminavano a gion untla polverieradi pioceto lando di «cicatrici che la guerFondazione Cariparma, ala divulgazione della storia e ele ne sociale, e per non ripetere ragici errori del Novecentos. Oggi presentiamo un lavor con carattere fortemente dire, ricerca delle fonti, doci-

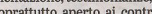
buti distorie memori arric giovane che non c'è più, ma clovane che non c'è più, $m$ crea importante su un pezzo di storia ancora da scrivere Prigionieri nei campialleatiè primoattodel progettodigita

di guerra, al quale sta ora lavorando Domenico Vitale. Allo stato attuale la ricerca ricorrec, facendone oggetto di un

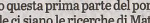
chimenti che possono arrivare dai cittadini. Lo faceiamo
valorizzando il lavoro di un

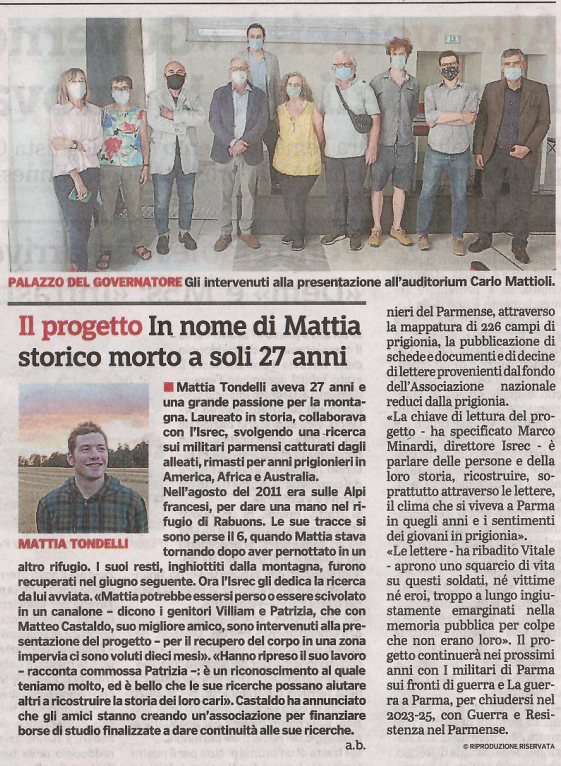

Fig. 1. Articolo della "Gazzetta di Parma" del 4 luglio 2020

\section{II portale Prigionieri nei campi alleati: una panoramica}

Nel corso della Seconda guerra mondiale furono fatti prigionieri 1.200 .000 militari italiani, 600.000 dei quali finirono in mano alleata ${ }^{2}$ [Conti 1986, 7]. Nel dopoguerra la questione di questi prigionieri ha subito una rimozione nella memoria pubblica e perfino la storiografia vi ha dedicato poca attenzione, rendendola di

II progetto, a cura di Marco Minardi e Domenico Vitale, ha avuto il sostegno della Regione Emilia-Romagna, della Provincia di Parma, del Comune di Parma e della Fondazione Cariparma. 
fatto uno dei temi meno noti della storia di quel conflitto. Negli ultimi anni il trend è però cambiato, con la pubblicazione di importanti studi in materia che hanno gettato luce sulle articolate vicende vissute da così tanti italiani. Essere prigionieri degli Alleati, in generale, significava godere di migliori condizioni rispetto a quelle degli Internati militari italiani (Imi) detenuti dai tedeschi anche se, nella maggior parte dei casi, la detenzione fu più lunga: in media di tre-quattro anni fino ad arrivare a sei anni di prigionia.

Se i grandi numeri e le macro-ricerche restituiscono un'immagine generale del fenomeno, sono spesso gli studi locali a permettere uno sguardo ravvicinato, capace di cogliere sfumature e significati altrimenti non evidenti. In questa direzione si pone il portale web Parma in tempo di guerra: Prigionieri nei campi alleati.

Grazie allo spoglio dell'archivio della federazione parmense dell'Associazione nazionale reduci della prigionia (Anrp) - conservato presso l'Istituto storico della Resistenza e dell'età contemporanea di Parma - si è potuto ricostruire la storia di 1.181 militari del parmense catturati dagli Alleati. L'archivio, infatti, conserva i fascicoli personali dei reduci, contenenti le informazioni anagrafiche e quelle relative alla prigionia.

I dati di questi 1.181 prigionieri sono stati caricati su una banca dati all'interno del portale. È stato inol-

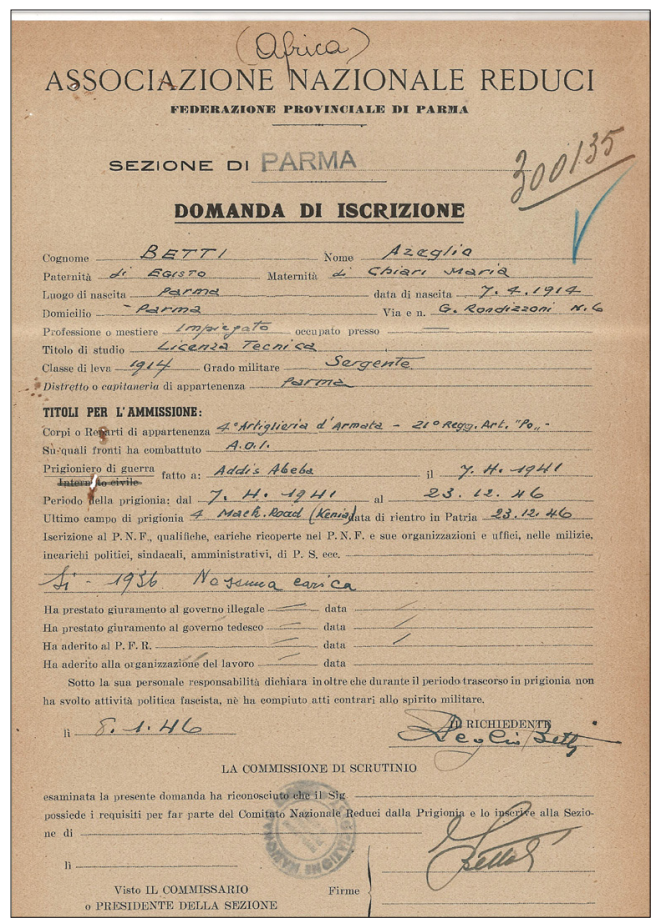

Fig. 2. Esempio di scheda del reduce della prigionia iscritto all'Arnp tre possibile individuare 226 luoghi di prigionia, sparsi per tutti i cinque continenti, consultabili attraverso una mappa interattiva. La maggior parte dei soldati del Parmense - circa un migliaio - fu catturata in Africa, la restante parte quasi tutta in Italia. Il 75\% fu detenuta dagli inglesi e internata per tre quarti nei vari dominions britannici, uno su quattro fu invece inviato in Gran Bretagna con l'obbiettivo di essere utilizzato come forza lavoro [Insolvibile 2012]. Il 13\% del totale dei prigionieri del Parmense fu de- 
tenuto dai francesi e, con alcune eccezioni, internato nelle colonie nordafricane, mentre il 12\% finì in mano statunitense e inviato per il 90\% negli Stati Uniti. Uno squarcio sull'esperienza di prigionia ci viene offerto dalle lettere, tante, conservate nell'archivio Anrp di Parma. Ogni ex prigioniero, infatti, al momento dell'iscrizione all'associazione doveva consegnare un documento che comprovasse la propria prigionia: nella maggior parte dei casi si trattava di lettere o cartoline inviate o ricevute al campo, conservate oggi nei fascicoli di ogni reduce. L'archivio - che contiene le schede di circa 3.000 reduci della prigionia (contando anche gli Imi e i soldati catturati in Russia) - ha quindi un patrimonio di più di un migliaio di lettere e cartoline, a cui si aggiunge altra documentazione di vario interesse (documenti di prigionia, certificati di vaccinazione, attestati ottenuti da parte alleata, ecc.). Una cinquantina di queste lettere sono state trascritte, caricate nel portale e ordinate per soggetto. Sono lettere che ci parlano dell'alimentazione, della vita nel campo di prigionia, del lavoro, della cooperazione con gli Alleati, dell'attesa del ritorno, di progetti, paure e speranze. Da queste si evincono anche le articolate e a volte opposte condizioni che questi prigionieri si trovavano a vivere in base a chi li detenesse, al campo di internamento e all'aver scelto se cooperare o meno con gli Alleati?

A questo proposito, prendiamo ad esempio due lettere: la prima scritta il 2 agosto 1943 da Giovanni Chiari ${ }^{4}-$ detenuto dagli statunitensi - e inviata alla propria famiglia dal campo di Como, Mississippi, dalla quale emerge una condizione molto positiva:

Carissimi $[\ldots]$ Vi dico che mi trovo in America in un paese che si chiama Como che si trova nel Mississippi. Il viaggio lo [sic] fatto abbastanza bene

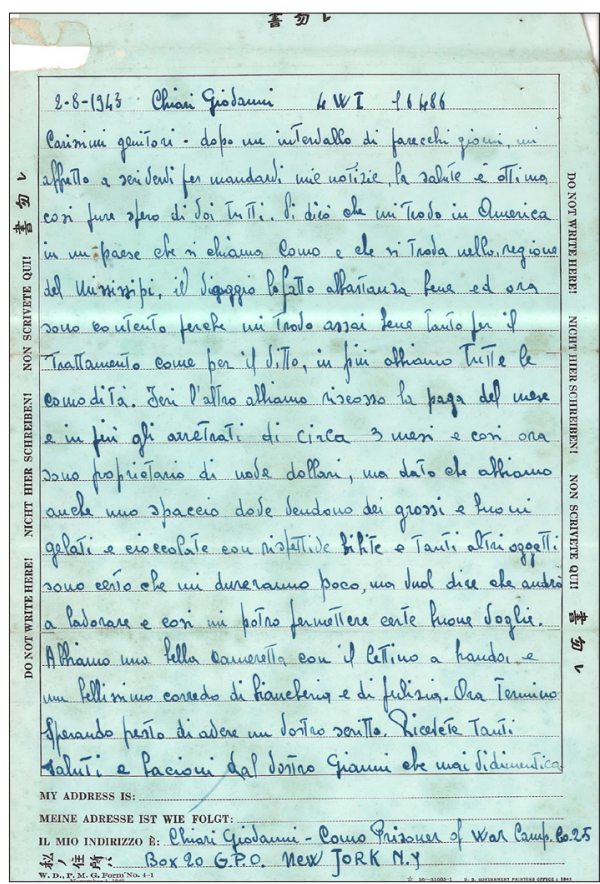

Fig. 3. Lettera inviata alla famiglia da Giovanni Chiari dal campo di Como (Mississippi, Stati Uniti) in data 2 agosto 1943 
ed ora sono contento perché mi trovo assai bene per il trattamento come per il vitto, in più abbiamo tutte le comodità. Ieri l'altro abbiamo riscosso la paga del mese e in più gli arretrati di circa 3 mesi e così ora sono proprietario di nove dollari, ma dato che abbiamo anche uno spaccio dove vendono grossi e buoni gelati e cioccolate con rispettive bibite e tanti oggetti sono certo che mi dureranno poco, ma vuol dire che andrò a lavorare e così mi potrò permettere certe buone voglie. Abbiamo una bella cameretta con il lettino a branda e un bellissimo corredo di biancheria e di pulizia.

Questo genere di lettere rappresentava anche un importante veicolo di propaganda statunitense: il racconto delle condizioni di prigionia del soldato, possiamo immaginare, letto da amici e familiari italiani oppressi dalle ristrettezze della guerra, doveva suscitare un misto tra invidia e gratitudine.

Di tenore completamente diverso è invece la seconda lettera, scritta da Italo Frambati ${ }^{5}$ il $1^{\circ}$ luglio 1947 dal campo inglese di Zonderwater, Sudafrica:

Per quanto sia rassegnato a questa dura vita, vi sono diversi momenti in cui questa mia rassegnazione è superata dallo sconforto della disperazione. Non potrebbe essere altrimenti dato la lunga monotonia del reticolato, e per di più, ad aggravare questa nostra terribile situazione è sopravvenuto quel terrificante spettro della fame quale pensa a diminuire quelle poche energie rimasteci dopo anni di prigionia. Giorno per giorno ci vengono razionati e diminuiti quei pochi alimenti quali sarebbe appena sufficienti per mantenerci in piedi.

A differenza della prima lettera, questa - scritta in un momento e in un contesto diverso - racconta una prigionia fatta di stenti e rassegnazione.

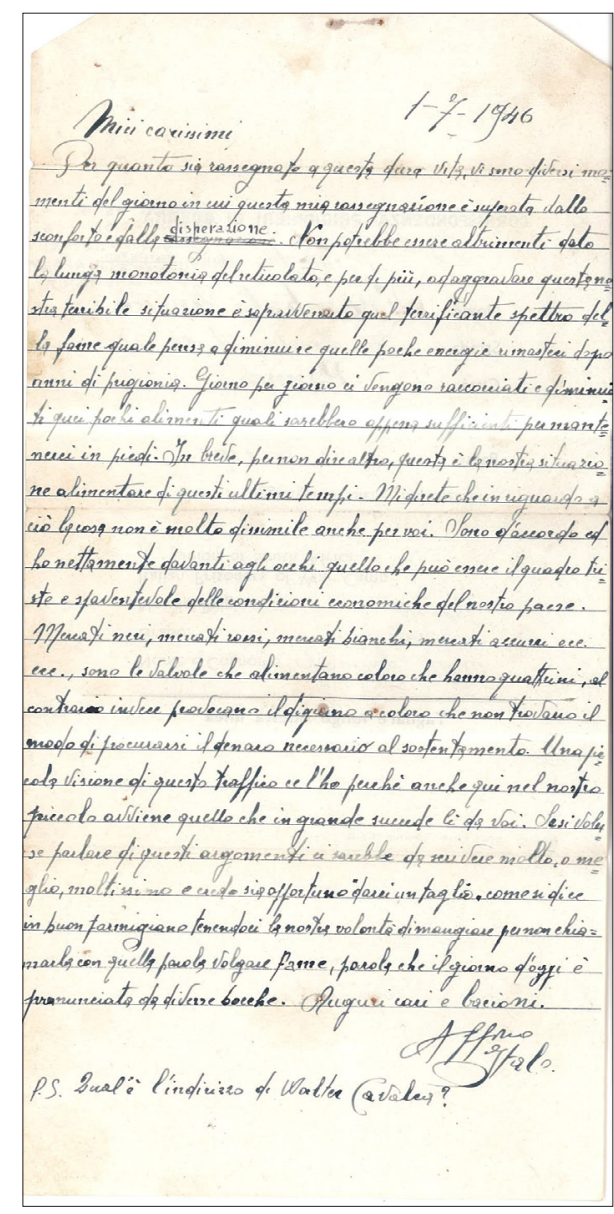

Fig. 4. Lettera inviata alla famiglia da Italo Frambati dal campo di Zonderwater (Sudafrica) in data $1^{\circ}$ luglio 1946 


\begin{abstract}
La sezione Prigionia del portale, infine, è riservata alle schede di approfondimento, dove si è cercato di tenere assieme la dimensione generale del tema intrecciandolo ai risultati e le riflessioni dei dati del Parmense. Queste schede sono, per la maggior parte, ricavate dalla tesi di laurea magistrale di Mattia Tondelli, giovane ricercatore scomparso tragicamente nel 2011, che per primo ha avviato questa ricerca e alla cui memoria è dedicato il portale ${ }^{6}$.
\end{abstract}

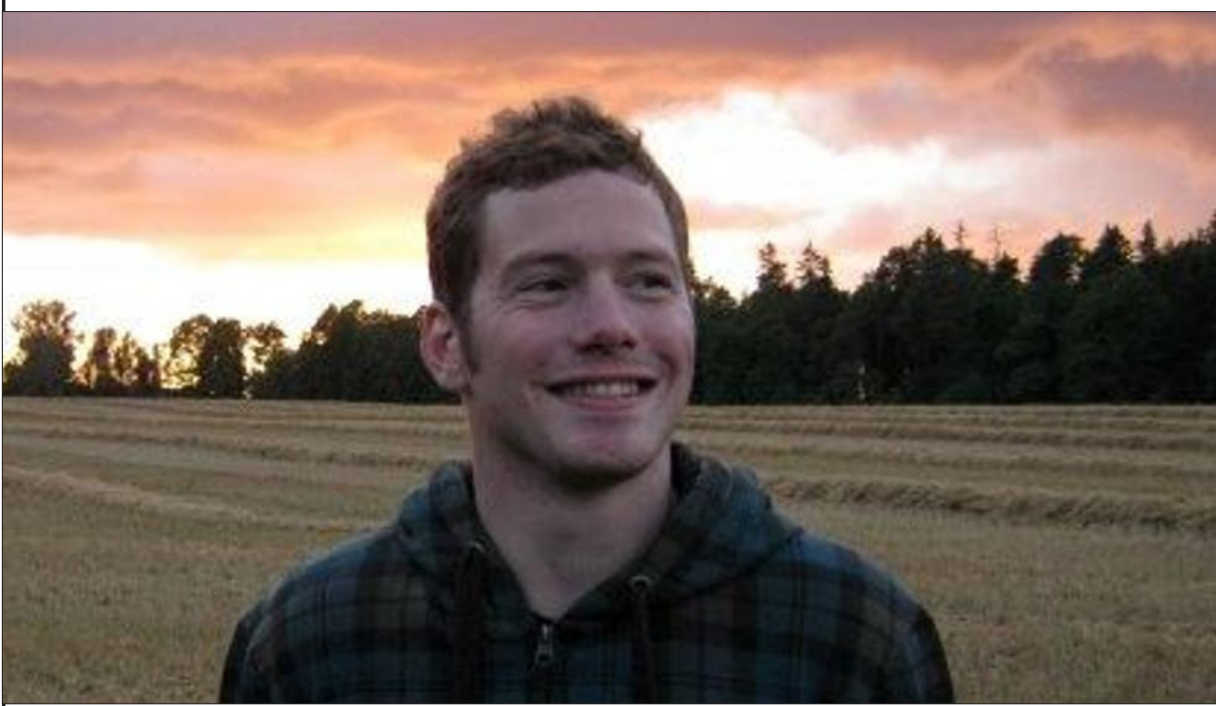

Fig. 5. Mattia Tondelli

\title{
3. Conclusioni
}

Il portale Prigionieri nei campi alleati è in costante aggiornamento, attraverso l'implementazione di contenuti e schede di approfondimento. Inoltre, la sua pubblicazione ha portato all'interessamento di alcuni parenti di prigionieri, dai quali riceviamo nuove informazioni e documenti. Attraverso contributi privati e nuove ricerche si intende, quindi, integrare la banca dati con nuovi nominativi, estendendola anche ai prigionieri catturati e detenuti dall'Unione Sovietica.

Uno degli sviluppi fondamentali sarà la realizzazione di percorsi didattici rivolti alle scuole del territorio, i cui risultati saranno caricati in una sezione apposita del portale. 
Nei prossimi mesi si prevede la pubblicazione dei successivi capitoli di Parma in tempo di guerra 1940-1945: al momento è in lavorazione Militari al fronte 19401943, un portale che racconterà - sempre attraverso l'uso di mappe, documenti, immagini e schede di approfondimento - l'esperienza al fronte dei soldati del Parmense dall'ingresso in guerra fino all'armistizio.

L'obbiettivo ambizioso è di giungere, in occasione dell'ottantesimo dell' 8 settembre 1943, al quarto portale dedicato alla Resistenza.

\section{Bibliografia}

Bersani F. 1997, I dimenticati: i prigionieri italiani in India: 1941-1946, Milano: Mursia

Bistarelli A. 2007, La storia del ritorno: i reduci italiani del secondo dopoguerra, Torino: Bollati Boringhieri

Carlesso L. 2009, Centomila prigionieri italiani in Sud Africa: il campo di Zonderwater, Ravenna: Longo

Conti F.G. 1986, I prigionieri di guerra italiani 1940-45, Bologna: il Mulino

De Prospo M. 2010, I prigionieri di guerra italiani negli Stati Uniti e il dilemma della cooperazione (1944-1946), "Diacronie: Studi di Storia Contemporanea", 1

Dubois C. 1989, Internés et prisonniers de guerre italiens dans le camps de l'empire français de 1940 a 1945, "Guerres mondiales et conflits contemporains: revue d'histoire", ottobre

Hall J. 1999, Private Memories, Public Perceptions: Italian prisoners of war in Northern New South Wales, "Limina", 5

Insolvibile I. 2012, Wops. I prigionieri in Gran Bretagna (1941-1946), Napoli: Edizioni scientifiche italiane

Moore B. e Fedorowich K. 2002, The British Empire and its Italian prisoners of war, 1940-1947, New York: Palgrave

Rochat G. 1987, Prigionia di guerra e internamento nell'esperienza dei soldati italiani, in Spostamenti di popolazione e deportazioni in Europa: 1939-1945, Bologna: Cappelli editore

\section{Risorse}

Parma in tempo di guerra 1940-1945

https://parmaintempodiguerra.it/

Parma in tempo di guerra: Prigionieri nei campi alleati https://prigionieri.parmaintempodiguerra.it/ 
